Arteterapia. Papeles de arteterapia y educación para inclusión social ISSN-e 1988-8309

http://dx.doi.org/10.5209/ARTE.57574

\title{
Mariposas. Transformación interior a través del Arte
}

\author{
Tania Ugena Candel $^{1}$, Ana Casla Puig ${ }^{2}$
}

Recibido: 25 de Julio de 2017 / Aceptado: 6 de septiembre de 2017

Resumen. Este artículo presenta al CMSc Centro Joven del Ayuntamiento de Madrid, lugar donde se está implementando el Taller MARIPOSAS. TRANSFORMACIÓN INTERIOR A TRAVÉS DEL ARTE. El texto recoge las diferentes fases de este proceso: la detección de la necesidad percibida por el equipo multidisciplinar, las reuniones de coordinación con las profesionales implicadas, la definición de objetivos y el diseño del Taller a partir del conocimiento de las características de las jóvenes, los criterios e instrumentos de evaluación y las conclusiones y prospectiva de esta investigación.

Palabras clave: trauma; cuerpo; autorregulación; autoeficacia; arteterapia.

\section{[en] Butterflies: Inner transformation through Art}

Abstract. This paper presents the CMSc Centro Joven of Madrid city hall, place where the workshop BUTTERFLIES. INNER TRANSFORMATION THROUGH ART is being implemented. The text comprehends the different stages of this process: the detection of the necessity noticed by the multidisciplinary team, the coordination meetings with the implicated professionals, the definition of objectives and the workshop design from the knowledge of the young women characteristics, the criteria and assessment resources and future-oriented reflection of this research.

Key words: Trauma; body; self-regulation; self-efficacy; art therapy

Sumario. 1. CMSc Centro Joven. 2. Respuesta a una necesidad detectada. 3. Fase de Diseño del Taller. 4. Fase de Implementación del Taller. 5. Características del grupo susceptible de participar en el Taller. 6. Fase de Desarrollo del Taller. 7. Fase de Evaluación del Taller. 8. Mujeres en danza para superar el trauma. 9. Conclusiones y prospectiva. 10. Bibliografía.

Cómo citar: Ugena Candel, T.; Casla Puig, A. (2017). Mariposas. Transformación interior a través del Arte, en Arteterapia. Papeles de arteterapia y educación para inclusión social 12, 233-242.

1 Arteterapeuta CMSc Centro Joven - Ayuntamiento de Madrid. Doctora en educación. Máster en Arteterapia. Licenciada en Antropología. Diplomada en Educación Social. Formación en Danza Clásica y Flamenco. Directora Girasol Arteterapia en Movimiento.

E-mail: arteterapia@girasolarteterapia.es

2 Psicóloga CMSc Centro Joven - Ayuntamiento de Madrid

Licenciada en Psicología. Máster en Psicoterapia de Tiempo Limitado y Psicología de la Salud. Máster en Habilidades del Terapeuta Cognitivo-Conductual.

E-mail: caslapa@madrid.es 


\section{CMSc Centro Joven}

El CMSc Centro Joven (en adelante CMScJ) es un centro público del Ayuntamiento de Madrid perteneciente al Organismo Autónomo Madrid Salud del Área de Gobierno de Salud, Seguridad y Emergencias.

$\mathrm{La}$ atención en el CMScJ es gratuita y confidencial, está dirigida a chicos y chicas de entre 12 y 24 años (aunque prioriza a los menores de 21 años). Esta atención la desarrolla un equipo multidisciplinar (medicina, enfermería, ginecología, psicología, arteterapia y trabajo social), especialmente formados en adolescentes y jóvenes.

El CMScJ brinda apoyo en la salud sexual y reproductiva (métodos anticonceptivos, píldora de anticoncepción de urgencia, test de embarazo, información sobre interrupción voluntaria del embarazo, consejo y prueba de VIH e ITS), asesoría sexual, ginecología de la adolescencia, asesoría de drogas, maternidad temprana, orientación emocional y familiar, consejo nutricional y educación para la salud.

Estas acciones se encuadran dentro de los siguientes programas marco de los Centros Municipales de Salud Comunitaria (CMSc): Promoción de la Salud Mental y Psicohigiene; Materno Infantil; Ámbito educativo; Salud Sexual y Reproductiva; Desigualdades Sociales.

Desde marzo de 2017 comienza el Programa de Arteterapia en el CMScJ con la convocatoria de la Beca de formación e investigación sobre la "Aplicación de metodologías artísticas al apoyo de los cuidados en la vida cotidiana de los barrios (arte y cuidados)", surgida gracias al impulso de Madrid Ciudad de los Cuidados.

Un modelo de ciudad promovido por el Ayuntamiento de Madrid que gira en torno a los cuidados de la ciudadanía para garantizar la cobertura de sus necesidades básicas de manera transversal y se articula en torno a cuatro ejes vertebradores: la salud y sus determinantes sociales, el barrio, la vida cotidiana y el cuidado colectivo.

\section{Respuesta a una necesidad detectada.}

MARIPOSAS. TRANSFORMACIÓN INTERIOR A TRAVÉS DELARTE, se plantea a partir de una necesidad detectada por el equipo multidisciplinar de CMScJ, donde vienen a consulta chicas jóvenes con situaciones traumáticas no resueltas. Refieren problemas sexuales derivados del trauma y dificultades importantes para el desarrollo de su vida cotidiana a todos los niveles (afectivo, social, relacional, cognitivo, físico...). Acuden a los servicios de asesoría sexual y de planificación familiar por dificultades en la vivencia de su sexualidad, empleo de métodos anticonceptivos y/o necesidad recurrente de anticoncepción de urgencia.

Un gran número de estas jóvenes vive en Casas de Acogida donde reciben ayuda psicológica individual por profesionales que, frecuentemente, transmiten al equipo del CMScJ la dificultad para que estas chicas se adhieran a sus tratamientos. Presentando conflictos visibles en estas áreas: desconfianza y angustia ante el afrontamiento de memorias traumáticas, dificultad para establecer relaciones y para pedir ayuda.

La razón de esta propuesta de acción desde un servicio de promoción de la salud comunitaria es ayudar a que estas chicas desarrollen una sensación de seguridad y capacidad en ellas mismas que, una vez obtenida, les permitirá afrontar sus recuer- 
dos traumáticos y desbloquear las intervenciones psicoterapeúticas individuales en las que están inmersas.

\section{Fase de Diseño del Taller}

En el Diseño propuesto para el Taller MARIPOSAS. TRANSFORMACIÓN INTERIOR A TRAVÉS DEL ARTE la principal herramienta es el cuerpo, por ser un vehículo fundamental para conectar con una misma. Aspecto que, junto a la planificación y estructura de las sesiones, la estrategia de captación del grupo, las reuniones con el equipo multidisciplinar y la metodología que se empleará en las sesiones pasa a desarrollarse a continuación.

Se trata de que vivan este Taller como un espacio seguro en el que no van a tener que afrontar aquello que les asusta sino descubrir y amplificar aquellos recursos que habitan en ellas; promoviendo su capacidad de agencia y empoderándolas como protagonistas de su propia vida. Lograr que puedan estar más centradas en su momento presente y en sus capacidades de autorregulación y planificación favorecerá una disminución de sus malestares, un aumento de su bienestar personal y de la percepción de autoeficacia.

Es fundamental realizar este trabajo desde CMScJ porque es un Centro de referencia para ellas, al que acuden por las consultas más arribas planteadas y es muy conveniente que lo contemplen como un espacio independiente de sus respectivas terapias, donde no van a tener que afrontar sus miedos sino descubrir y amplificar sus fortalezas.

La idea de trabajar desde el arteterapia es importante para que puedan diferenciar este proceso de otras experiencias terapéuticas que hayan podido tener antes, donde se priorice la sensación de disfrute y se fomenten emociones como la alegría, el juego, el compañerismo, la curiosidad y desde ahí puedan acercarse al descubrimiento de partes más sanas de sí mismas.

Este Taller puede ayudarlas a:

- Recuperar el vínculo con su cuerpo (la conciencia corporal es de vital importancia para poder conectar con el yo y con el ahora).

- Ser conscientes del presente sin miedo a sus propias sensaciones y emociones, al aunar cuerpo y mente.

- Vivir sus emociones como un foco de información que les señale sus necesidades para poder darles respuesta.

- Aumentar sus estrategias de autorregulación.

- Desarrollar respuestas más flexibles y adaptadas.

- Formar parte de un grupo que actúa como fuente de seguridad y apoyo; y que favorece la participación activa de las chicas.

- Utilizar las estrategias aprendidas para afrontar sus relaciones satisfactoriamente.

- Contar con un equipo multidisciplinar formado por las profesionales del Programa de Salud Sexual y Reproductiva y del Programa de Promoción de la Salud Mental y Psicohigiene y Arteterapia del CMScJ, en colaboración con las psicólogas que realizan la terapia individual de estas jóvenes en diferentes recursos. 


\section{Fase de Implementación del Taller}

En esta fase se están llevando a cabo diferentes acciones. Son fundamentales las reuniones de coordinación con las profesionales sanitarias que detectan en consulta los síntomas limitantes que manifiestan las jóvenes y que, en muchas ocasiones, bloquean cualquier tipo de avance en cuestiones relacionadas con su sexualidad y sus relaciones.

Por otra parte, la comunicación con la psicóloga referente de la Casa de Acogida donde residen estas chicas es vital a lo largo de todo el proceso. La derivación a la Asesoría Sexual de CMScJ y la devolución de las profesionales sanitarias de las situaciones percibidas generó la necesidad de crear un espacio donde dar respuestas a estas necesidades, como ya ha quedado reflejado en la fase de Diseño del Taller. Las reuniones posteriores para informar del mismo a la psicóloga de referencia de la Casa de Acogida fueron muy útiles, tanto para ajustar la propuesta de las sesiones como para definir el perfil de grupo más idóneo; además de para concretar la frecuencia de los encuentros con el propósito de realizar un seguimiento continuado de cada una de las participantes y de la evolución del grupo.

Entre agosto y septiembre está previsto iniciar el contacto con todas las jóvenes mediante una entrevista inicial e individual de cara a generar una vinculación positiva con las responsables del Taller, explicarles los objetivos, la metodología y la temporización del mismo.

Por la vinculación existente de las jóvenes con la profesional sanitaria referente de la Asesoría Sexual ${ }^{3}$, se ve pertinente que sea el enlace entre ellas y las responsables del Taller. La psicóloga de la Casa de Acogida manifiesta su satisfacción con el trabajo que se realiza en la Asesoría de CMScJ. Percibe que las jóvenes a las que deriva se sienten especialmente cuidadas, valorando la profesionalidad y la especialización en esa etapa de la vida, gracias a la cual ellas pueden explicar cómo ha sido su sexualidad y cómo quieren que sea en el futuro.

\section{Características del grupo susceptible de participar en el Taller}

La reunión con la psicóloga de la Casa de Acogida sirvió para clarificar los criterios de derivación y la situación actual de estas jóvenes, así como el funcionamiento interno del recurso en el que residen.

Es un Centro para mujeres de 18 a 25 años que han experimentado violencia intrafamiliar. Su estancia en el mismo es voluntaria y pueden llegar derivadas de Servicios Sociales, Centros de menores al llegar a la mayoría de edad o cualquier otro recurso que detecte esta situación.

La Casa de Acogida proporciona una intervención integral y se coordina con distintos dispositivos como CMScJ, SERMAS, Hospital Gregorio Marañón... Su objetivo es trabajar la autonomía, el posicionamiento en la realidad y el trauma. Para lograr estos objetivos las chicas pueden permanecer en la Casa de Acogida un máximo de dos años, con posibilidad de prórroga de seis meses.

Al ser tiempo insuficiente dada la problemática que estas jóvenes presentan (desregulación y desorientación interna, dificultad para establecer vínculos, frustración

María Isabel Santamaría Sánchez. Médico y sexóloga experta en salud sexual y reproductiva del CMScJ. 
acumulada, visión negativa de sí mismas y de su futuro), su proceso de recuperación y de autonomía lo empiezan a trabajar simultáneamente casi desde su llegada al Centro. En este sentido, las profesionales sienten una "espada de Damocles" sobre sus intervenciones, en el intento de respetar el tiempo de recuperación que cada una necesita y la inevitabilidad del cumplimiento de los plazos de estancia marcados. Una situación que aún se complica más cuando ingresan sin diagnóstico de salud mental, sin permiso de residencia, sin certificado de minusvalía...

Hay que tener en cuenta que este es el último recurso de la red que puede prestar atención a estas jóvenes, por ello es tan necesario que sea lo más eficaz posible. El objetivo una vez que finaliza su estancia en él es conseguir una vida autónoma, de no haberse logrado, su perspectiva es de una grave situación de indefensión y vulnerabilidad. Desde la Casa de Acogida intentan mitigar en lo posible este escenario ofreciéndolas pisos tutelados en casos de inteligencia límite, tramitación de la renta activa de inserción, programas para personas sin techo y procurando su mantenimiento en redes de salud.

El perfil de estas jóvenes es diverso pero tiene en común que todas tienen una historia de abandono, malos tratos y abusos desde la infancia. Cada una de ellas ha desarrollado sus propias estrategias para afrontar, o no, estas vivencias y continuar con su día a día.

Las experiencias traumáticas experimentadas han sido tan abrumadoras que han mermado la confianza en sí mismas hasta extremos muy paralizantes tanto en su vida diaria, en el afrontamiento de situaciones nuevas, como en su propio proceso de recuperación y procesamiento del trauma con una escasa percepción de autoeficacia.

En relación al cuerpo, principal herramienta de trabajo en este Taller, estas jóvenes oscilan entre dos extremos: alerta-rigidez y desconexión-laxitud.

En los casos de alerta-rigidez refieren dificultades para conciliar el sueño, alteración de la conducta alimentaria y sobreactivación de las respuestas defensivas manifestada en ojos muy abiertos, postura corporal tensa y sentidos vigilantes. En los casos de desconexión-laxitud, presentan movimientos mínimos, retraídos, cerrados y desconectados del momento presente. Miradas vacías, postura corporal hundida y sentidos anestesiados.

En ambos casos la respiración se resiente y sus respuestas están adaptadas a las situaciones traumáticas pasadas lo que les dificulta conectar con su presente. Puesto que la forma de lograr esta conexión es a través del cuerpo, es vital que descubran cómo es su respiración, dónde están a nivel corporal y postural, se den cuenta de si miran o no a los ojos, perciban lo tensas o relajadas que están y de qué forma vinculan con los otros y con su entorno.

Como ya se ha mencionado anteriormente el tiempo es una variable ineludible, por lo que para las profesionales de la Casa de Acogida es necesario que las jóvenes sean capaces de iniciar su tratamiento terapéutico del trauma lo antes posible. En este sentido el Taller MARIPOSAS. TRANSFORMACIÓN INTERIOR A TRAVÉS DEL ARTE es fundamental ya que apoya la toma de conciencia del propio yo, conectando cuerpo y mente y visibilizando los recursos que las participantes infravaloraban o creían no tener. 


\section{Fase de Desarrollo del Taller}

Se ha acordado con la psicóloga referente de la Casa de Acogida que las sesiones del Taller sean semanales y tengan una duración de dos horas. Se prevé que el trabajo va a ser lento, razón por la que no se ha establecido un número de sesiones determinado para evitar esa "espada de Damocles" que entorpece el respeto al ritmo de evolución que necesita cada participante.

Las situaciones traumáticas vivenciadas dificultan la confianza, el control, la orientación y la seguridad en sí mismas y en el entorno. Por este motivo, es fundamental que exista la certeza de que el compromiso de las profesionales se va a mantener en el tiempo y que el grupo va a ir avanzando en función de sus posibilidades, siempre dentro del margen de seguridad que ellas requieran.

Se va a consensuar con las jóvenes que deseen participar, si el horario del Taller será de mañana o de tarde, de forma que puedan compaginarlo con sus otras actividades y facilitar su presencia.

Cómo se ha planteado más arriba, el trabajo que se va a proponer va a ser fundamentalmente corporal y va a estar centrado en los recursos de cada participante para fomentar su bienestar y su autorregulación. En este sentido son imprescindibles las dinámicas que les ayudan a conectar con su cuerpo y a tomar conciencia de qué forma pueden regularse a través de esta conexión.

Como argumenta Bessel Van der Kolk "Las huellas del pasado pueden transformarse teniendo experiencias físicas que contradigan directamente la impotencia, la rabia y el colapso que forman parte del trauma, recuperando así el autocontrol" (Bessel Van der Kolk, 2015, p.4).

Para poder realizar este trabajo es fundamental que las participantes tengan una situación de seguridad objetiva que les permita diferenciar las vivencias pasadas de las presentes (actualmente, esta seguridad les viene dada por su estancia en la Casa de Acogida).

Es de vital importancia que ellas aprendan a reconocer esta sensación de seguridad en distintos espacios y acompañadas de diferentes personas para poder extrapolarla a otros ámbitos de su vida y comprender que la seguridad reside dentro de ellas mismas y no necesitan salir fuera a buscarla. La forma de detectar esta seguridad es a través de sensaciones corporales diversas que el Taller les va a posibilitar experimentar lúdica y creativamente.

Siguiendo a Ogden, Minton, y Pain:

La estimulación del sistema de acción responsable del juego y de las correspondientes emociones de diversión y placer son especialmente importantes en la terapia con los pacientes traumatizados que habitualmente son incapaces de emprender conductas lúdicas (Ogden, Minton, y Pain 2009, p.225)

En este sentido es enriquecedor el empleo del arteterapia porque abre un abanico de posibilidades en lo que respecta al juego como promotor de sensaciones placenteras que ayuden a las participantes a conectar con su presente y a vivirlo como gratificante y potencialmente motivador, alejándolas de una visión negativa y amenazante.

Por ello es fundamental construir un encuadre positivo donde cada participante pueda fluir en su propio movimiento, a fin de que diga lo que quiera decir, esté como quiera estar y pueda ser lo que es (Maslow, 1971). Que puedan crear libremente y 
sentir que el humor y el bien-estar están siempre presentes (Acouturier et al., 1985), requiere de una cierta continuidad con las profesionales, el tiempo, el espacio y los materiales (Panhofer, 2005).

Como la acción artística y creativa, rehace a quien la hace (Eisner, 1972), el objetivo del Taller es que estas jóvenes puedan llevar una vida personal satisfactoria y socialmente constructiva. Por todo lo cual, se considera el Taller un espacio y un tiempo para darse, para estar, para ser, que posibilita conectar con el ritmo personal a través de las artes y de la imaginación.

"El sello indicativo del sistema de acción responsable del juego es la risa, que favorece el apego y los lazos sociales" (Panksepp en Ogden, Minton, y Pain 2009, p.224). Lazos sociales que se van a fomentar en el grupo durante las sesiones del Taller, así como una visión diferente de ellas mismas y de sus relaciones fuera del entorno de la convivencia en la Casa de Acogida. Esto las puede ayudar a descubrir aspectos nuevos de sus compañeras que desconocían e incluso a superar y resolver conflictos. Adquiriendo un mayor manejo para establecer vínculos seguros y relaciones más sanas.

Una de las formas de trabajar la cohesión grupal va a ser a través de la sintonía rítmica con las participantes, empleando movimientos simples y sincronizados rítmicamente que generen en ellas estados corporales más regulados y diferentes a los que suelen presentar.

La danza, al igual que el movimiento, ayuda a tomar conciencia de cómo se involucra el cuerpo en la acción. Lo que permite extrapolar las sensaciones que se tienen bailando a otras situaciones vitales (Rodríguez en Panhofer, 2005).

Al bailar se utilizan muchas acciones corporales que cumplen cotidianamente con fines prácticos. Ayudando a recuperar la sensación de la niña que juega y que, sin necesidad de público, realiza diversas secuencias de combinaciones de esfuerzo o forma y repite diferentes frases rítmicas por puro placer (Le Du, 1976; Laban, 1975).

Además de la danza se explorarán otros lenguajes artísticos en los que el cuerpo y las emociones tienen un papel fundamental como el teatro, las artes plásticas y visuales o la música. Todo ello unido a la contención y el apoyo del grupo facilitará el descubrimiento de los recursos personales de cada una de las participantes y a tomar conciencia de su potencial individual y grupal.

\section{Fase de Evaluación del Taller}

La Evaluación va a ser continua a través de registros de observación individual y grupal, cuaderno de campo y registro visual de cada una de las sesiones.

El contenido de los registros de observación individual focaliza en las características corporales de hiperactivación e hipoactivación de las que se ha hablado anteriormente, y tienen una triple función: conocer la evolución de las participantes, adaptar las sesiones a esta evolución y poder transmitir y contrastar con ellas esta información. El objeto de esta última función es ayudarlas a tomar conciencia de su cuerpo y a ser conscientes de su proceso.

Es fundamental la triangulación con las profesionales responsables del Taller, la psicóloga con la que realizan el tratamiento individual en su Casa de Acogida y la referente del Programa de Salud Sexual y Reproductiva que trabaja en la Asesoría 
Sexual del CMScJ sus problemas relacionados con la sexualidad. Se pretende realizar reuniones periódicas para transmitir al equipo los aspectos más destacables del proceso de las jóvenes en el Taller, a fin de que puedan facilitar la acción de las otras profesionales en sus respectivas intervenciones y recibir su feed-back.

De igual forma, son primordiales las reuniones de devolución con las personas integrantes del Proyecto de Investigación I+D: ALETHEIA. ARTE, ARTETERAPIA, TRAUMA Y MEMORIA EMOCIONAL del grupo de investigación EARTDI, grupo de investigación consolidado (ref. 941035) en la Facultad de Educación de la Universidad Complutense de Madrid. Estos encuentros ayudan a dar a conocer esta experiencia, a conocer otras y a enriquecerlas con nuevas aportaciones.

\section{Mujeres en danza para superar el trauma}

Desde la prehistoria la danza ha sido empleada para afirmar al ser humano y su lugar en el mundo, siendo de gran importancia en sus ritos de paso y en momentos significativos de su vida.

Esta función de la danza fue denostada en mayor o menor medida en determinados momentos de la historia a causa de factores religiosos, culturales, políticos y sociales. Sin embargo, nunca perdió su potencial de sanación y la posibilidad de fortalecer la psique gracias al cuerpo en movimiento.

Estas danzas espontáneas surgidas del anhelo de vivir, del tesón y la persistencia por superar las adversidades, han sido recogidas en coreografías que muestran dramas humanos en un intento de canalizar el dolor y el sufrimiento desde el sentido estético. Coreógrafas y bailarinas como Marta Graham, Angna Enters, Helen Tamiris, Ruth Page, Anna Sokolow, Sophie Maslow, Ida Soyer, Lily Mehlman, entre muchas otras, han puesto en escena los traumas derivados de la violencia y sus consecuencias.

Años más tarde estas autoras inspiraron a las danza movimiento terapeutas, que comprobaron en sus talleres cómo la danza ayudaba a las personas participantes a reconocer, expresar y resolver su sufrimiento. Un sufrimiento que hasta ese momento lo habían experimentado desconectado del cuerpo o de la mente y que, con la práctica de la danza, consiguieron percibir globalmente.

En experiencias de abordaje del trauma, en general, y con mujeres, en particular, se plantea la importancia de trabajar desde el cuerpo y, al mismo tiempo, la dificultad para establecer vías de acceso a éste.

Una de las formas para vencer este obstáculo es emplear el dibujo en las primeras sesiones como una forma de expresión menos amenazante y más contenedora. Hay evidencias que demuestran que los dibujos van adquiriendo mayor complejidad y riqueza simbólica conforme avanzan las sesiones y aumenta la conexión con el propio cuerpo (Lewis, 1999; Figueira en Panhofer, 2005; Levy, 1995).

Los objetos como telas, globos, pelotas, cuerdas, lanas..., empleados en un contexto lúdico también actúan como facilitadores para vencer las resistencias iniciales de conectar con el cuerpo, dando lugar a movimientos más libres y espontáneos.

Otro recurso fundamental es el uso de imágenes y metáforas que generan emociones, sensaciones y movimientos particulares para cada una de las mujeres que participan en el Taller. 
En todo este proceso es fundamental generar sensación de seguridad y aumentar la capacidad de autorregulación de las participantes. Para ello son muy útiles las dinámicas de alineación de la columna, ya que la postura o forma de sostener el cuerpo expresa los sentimientos y el modo de afrontar la vida; las dinámicas de enraizamiento que aumentan la capacidad de dirigir la energía hacia el suelo para obtener seguridad y confianza; y las dinámicas de respiración que ayudan a regular el estado de ánimo.

Dada la eficacia demostrada de las herramientas descritas, éstas se han tenido en cuenta en la fase de diseño más arriba desarrollada.

\section{Conclusiones y prospectiva}

Los resultados esperados en el Taller MARIPOSAS. TRANSFORMACIÓN INTERIOR A TRAVÉS DEL ARTE son diversos y pueden englobarse en distintos niveles.

Por una parte, que las participantes logren tomar conciencia de sus sensaciones corporales a través del movimiento (centrarse, arraigarse, respirar, alinearse), les genera una sensación de estabilidad interior física, mental y emocional a través de la cual pueden descubrir sus recursos de autorregulación, seguridad y confianza en sí mismas y en sus capacidades. La conexión con estos recursos revertirá positivamente en su capacidad de agencia ante sus respectivas terapias individuales, sus relaciones y su vida actual.

La vinculación generada en el grupo favorece la creación de redes de apoyo, entre iguales y con diferentes profesionales y recursos, que actúan como un activo de salud al prevenir y/o mitigar el aislamiento social. Por otro lado, sentirse incluidas y valoradas en estas redes les ayuda a modificar la percepción que tienen sobre los vínculos, transformando el miedo, el rechazo o la dependencia en seguridad, aceptación e interdependencia.

La oportunidad de encuentro con otras jóvenes que han experimentado situaciones similares les permite ampliar la mirada con la que se perciben a sí mismas y a su historia de vida, nutriéndola de otras experiencias o modos de afrontamiento más adaptativos, flexibles y eficaces.

Estos encuentros se ven propiciados al trabajar coordinadamente con otras entidades que acompañan a estas jóvenes. Coordinación que, además, crea sinergias que aumentan la eficacia y la optimación de los recursos, así como el enriquecimiento de los equipos interdisciplinares.

Actualmente los Servicios de Salud detectan una variada sintomatología (física, mental, emocional), sin causa física evidente y con diagnóstico múltiple, que refleja maneras de actuar o de posicionarse ante la vida con origen en traumas de distinta gravedad no resueltos. Este Taller da respuesta a esta problemática al ser una herramienta de trabajo valiosa que permite adquirir mayor nivel de competencia y seguridad para afrontar retos.

El hecho de nacer mujer en nuestra sociedad supone una condición de vulnerabilidad que, añadida a las circunstancias vitales de estas jóvenes, las convierte en un sector de población de atención prioritaria. Por ello, este Taller pretende favorecer el disfrute de una vida con oportunidades de salud, recursos accesibles y procesos inclusivos. 
En un futuro sería deseable que el trabajo que se viene desarrollando CMScJ y que ha sido expuesto en este artículo pueda ser extrapolado a otros Centros Municipales de Salud Comunitaria (CMSc) para favorecer el aumento del bienestar y el acceso a la salud de toda la ciudadanía.

\section{Bibliografía}

Amighi, J., Loman, S., Lewis, P., et al. (1999). The meaning of movement developmental and clinical perspectives of the Kestenberg movement profile. Amsterdam: Gordon and Breach Publishers.

Acouturier, B., Darrault, I. y Empinet, J.L. (1985). La práctica psicomotriz. Reeducación y terapia. Barcelona: Editorial científico médica.

Bessel Van der Kolk, M.D. (2015). El cuerpo lleva la cuenta. Cerebro, mente y cuerpo en la superación del trauma. Barcelona: Eleftheria.

Eisner, E. (1972). Educar la visión artística. Barcelona: Paidós.

Laban, R. (1975). Danza educativa moderna ( $3^{\mathrm{a}}$ ed.). Barcelona: Paidós.

Le Du, J. (1976). El cuerpo hablado. Psicoanálisis de la expresión corporal. Barcelona: Paidós.

Levy, F.J., Fried, J.P. y Leventhal, F. (Eds.) (1995). Dance and other expressive arts therapies: when words are not enough. Londres: Routledge.

Ogden, P., Minton, K. y Pain, C. (2009). El trauma y el cuerpo: un modelo sensoriomotriz de psicoterapia. Bilbao: Desclée de Brouwer.

Ogden, P. y Fisher. J. (2016). Psicoterapia sensoriomotriz. Intervenciones para el trauma y el apego. Bilbao: Desclée de Brouwer.

Panhofer, H. (Comp.)(2005). El cuerpo en psicoterapia. Teoría y práctica de la danza movimiento terapia. Barcelona: Gedisa.

Reca, M. (2011). Tortura y trauma. Danza/movimiento terapia en la reconstrucción del mundo de sobrevivientes de tortura por causas políticas. Buenos Aires: Biblos. 